\title{
Odd Generalized Exponential Flexible Weibull Extension Distribution
}

\author{
Abdelfattah Mustafa \\ Mathematics Department, Faculty of Science, Mansoura University, \\ Mansoura, Egypt \\ abdelfatah_mustafa@yahoo.com \\ Beih S. El-Desouky \\ Mathematics Department, Faculty of Science, Mansoura University, \\ Mansoura, Egypt \\ b_desouky@yahoo.com \\ Shamsan AL-Garash \\ Mathematics Department, Faculty of Science, Mansoura University, \\ Mansoura, Egypt \\ shamsan_algarash@hotmail.com
}

Received 21 December 2016

Accepted 29 December 2017

\begin{abstract}
In this article we introduce a new four - parameters model called the odd generalized exponential flexible Weibull extension (OGE-FWE) distribution which exhibits bathtub-shaped hazard rate. Some of it's statistical properties are obtained including ordinary and incomplete moments, quantile and mode, the moment generating functions, reliability and order statistics. The method of maximum likelihood is used for estimating the model parameters and the observed Fisher's information matrix is given. Moreover, we give the advantage of the OGE-FWE distribution by an application using real data.
\end{abstract}

Keywords: Odd Generalized Exponential Family; Flexible Weibull Extension distribution; Generalized Weibull; Odd Generalized Exponential Flexible Weibull distribution; Maximum likelihood estimation.

2000 Mathematics Subject Classification: 90B25, 62N05, 62P30

\section{Introduction}

The Weibull distribution is a highly known distribution due to its utility in modelling lifetime data where the hazard rate function is monotone, [27]. Recently new classes of distributions were proposed based on modifications of the Weibull distribution to provide a good fit to data set with bathtub hazard failure rate, see [25]. Among of these, Exponentiated Weibull family, [14], Modified Weibull distribution, [11, 19], Beta-Weibull distribution, [8], A flexible Weibull extension, [4], Extended flexible Weibull, [4], Generalized modified Weibull distribution, [5], Kumaraswamy Weibull distribution, [6], Beta modified Weibull distribution, [16,22], Beta generalized Weibull distribution, [23], 
A new modified weibull distribution, [2] and Exponentiated modified Weibull extension distribution, [20], among others. A good review of these models is presented in [3, 15, 17].

The flexible Weibull extension (FWE) distribution, [4] has many applications in life testing experiments, reliability analysis, applied statistics and clinical studies. For more details on this distribution, see [4].

A random variable $X$ is said to have the Flexible Weibull Extension (FWE) distribution with parameters $\alpha, \beta>0$ if it's probability density function (pdf) is given by

$$
g(x)=\left(\alpha+\frac{\beta}{x^{2}}\right) \exp \left\{\alpha x-\frac{\beta}{x}-e^{\alpha x-\frac{\beta}{x}}\right\}, x>0,
$$

while the cumulative distribution function (cdf) is given by

$$
G(x)=1-\exp \left\{-e^{\alpha x-\frac{\beta}{x}}\right\}, x>0 .
$$

The survival function is given by the equation

$$
S(x)=1-G(x)=\exp \left\{-e^{\alpha x-\frac{\beta}{x}}\right\}, x>0,
$$

and the hazard rate function is

$$
h(x)=\left(\alpha+\frac{\beta}{x^{2}}\right) e^{\alpha x-\frac{\beta}{x}} .
$$

Gupta and Kundu [9] proposed a generalization of the exponential distribution named as Generalized Exponential (GE) distribution. The GE distribution with parameters $\vartheta, \gamma>0$, has the following distribution function

$$
F(x ; \vartheta, \gamma)=\left(1-e^{-\vartheta x}\right)^{\gamma}, x>0, \vartheta>0, \gamma>0
$$

Recently, a new class of univariate continuous distributions named as the odd generalized exponential (OGE) class introduced in [7,24]. This class is flexible because of the hazard rate shapes could be increasing, decreasing, bathtub and upside down bathtub. The odd generalized exponential (OGE) class is defined as follows.

If $G(x), x>0$ is cumulative distribution function (cdf) of a random variable $X$, then the corresponding survival function is $\bar{G}(x)=1-G(x)$ and the probability density function is $g(x)$, then we define the cdf of the OGE class by replacing $x$ in the distribution function of generalized exponential (GE) distribution given in equation (1.5) by $\frac{G(x)}{\bar{G}(x)}$ leading to

$$
F(x ; \vartheta, \gamma)=\left[1-\exp \left\{-\vartheta \frac{G(x)}{\bar{G}(x)}\right\}\right]^{\gamma}, x>0, \vartheta>0, \gamma>0 .
$$

The probability density function corresponding to (1.6) is given by

$$
f(x ; \vartheta, \gamma)=\frac{\vartheta \gamma g(x)}{\bar{G}(x)^{2}} \exp \left\{-\vartheta \frac{G(x)}{\bar{G}(x)}\right\}\left[1-\exp \left\{-\vartheta \frac{G(x)}{\bar{G}(x)}\right\}\right]^{\gamma-1}
$$

where $x>0, \vartheta>0, \gamma>0$. In this article we present a new distribution depending on flexible Weibull extension distribution referred to as the odd generalized exponential flexible Weibull extension 
(OGE-FWE) distribution by using the class of univariate distributions defined above.

This paper can be organized as follows, we define the cumulative, density and hazard functions of the odd generalized exponential flexible Weibull extension (OGE-FWE) distribution in Section 2. In Sections 3, we present some statistical properties including, quantile function and median, the mode, $r$ th moment, skewness and kurtosis. In Sections 4, we introduce the moment generating function. The distribution of the order statistics is expressed in Section 5. The maximum likelihood estimation of the parameters is determined in Section 6. We use real data sets and analyzed it by an application in Section 7 and the results are compared with existing distributions. Finally, we present a conclusion in Section 8.

\section{The Odd Generalized Exponential Flexible Weibull Extension Distribution}

In this section we studied the four parameters odd generalized exponential flexible Weibull extension OGE-FWE $(\vartheta, \gamma, \alpha, \beta)$ distribution. Using $G(x)$ from Eq. (1.2) and $g(x)$ from Eq. (1.1) to obtain the cdf and pdf of Eqs. (1.6) and (1.7), respectively. The cumulative distribution function cdf of the odd generalized exponential flexible Weibull extension distribution (OGE-FWE) is given by

$$
F(x ; \vartheta, \gamma, \alpha, \beta)=\left[1-e^{-\vartheta\left(e^{e^{\alpha x-\frac{\beta}{x}}}-1\right)}\right]^{\gamma}, x>0, \vartheta, \gamma, \alpha, \beta>0
$$

The pdf corresponding to Eq. (2.1) is given by

$$
f(x ; \vartheta, \gamma, \alpha, \beta)=\vartheta \gamma\left(\alpha+\frac{\beta}{x^{2}}\right) e^{\alpha x-\frac{\beta}{x}+e^{\alpha x-\frac{\beta}{x}}} e^{-\vartheta\left(e^{e^{\alpha x-\frac{\beta}{x}}}-1\right)}\left[1-e^{-\vartheta\left(e^{e^{\alpha x-\frac{\beta}{x}}}-1\right)}\right]^{\gamma-1}
$$

where $x>0$ and, $\alpha, \beta>0$ are two additional shape parameters.

The survival function $S(x)$, hazard rate function $h(x)$ and reversed hazard rate function $r(x)$ of $X \sim$ OGE-FWE $(\vartheta, \gamma, \alpha, \beta)$ are given by

$$
\begin{aligned}
& S(x ; \vartheta, \gamma, \alpha, \beta)=1-\left[1-e^{-\vartheta\left(e^{\alpha x-\frac{\beta}{x}}-1\right)}\right]^{\gamma}, x>0,
\end{aligned}
$$

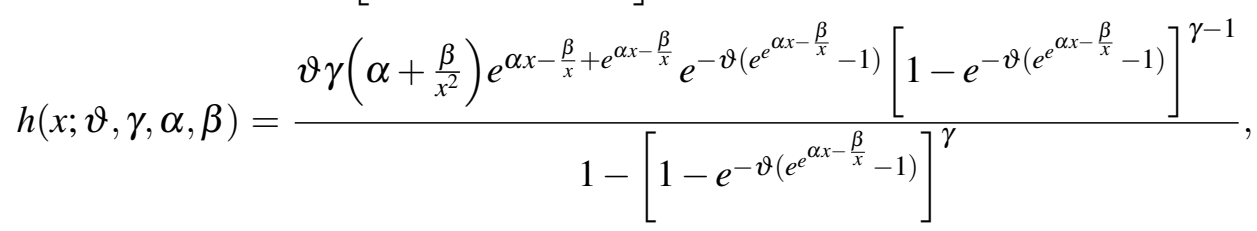

$$
\begin{aligned}
& r(x ; \vartheta, \gamma, \alpha, \beta)=\frac{\vartheta \gamma\left(\alpha+\frac{\beta}{x^{2}}\right) e^{\alpha x-\frac{\beta}{x}+e^{\alpha x-\frac{\beta}{x}}} e^{-\vartheta\left(e^{\alpha x-\frac{\beta}{x}}-1\right)}}{\left.1-e^{-\vartheta\left(e^{\alpha x-\frac{\beta}{x}}\right.}-1\right)},
\end{aligned}
$$

respectively, $x>0$ and $\vartheta, \gamma, \alpha, \beta>0$.

Figures (1-3) display the cdf, pdf, survival, hazard rate and reversed hazard rate function of the OGE-FWE $(\vartheta, \gamma, \alpha, \beta)$ distribution for some parameter values. 


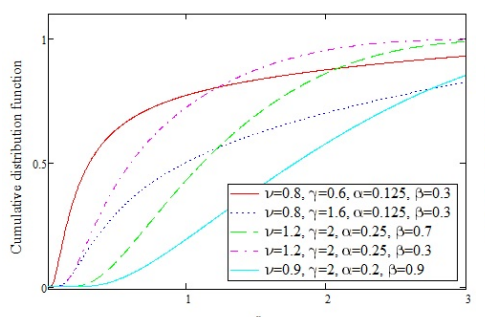

(a) cdf

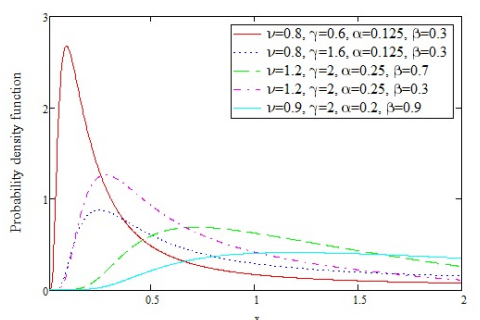

(b) pdf

Fig. 1. The cdf and pdf of the OGE-FWE for different values of parameters.

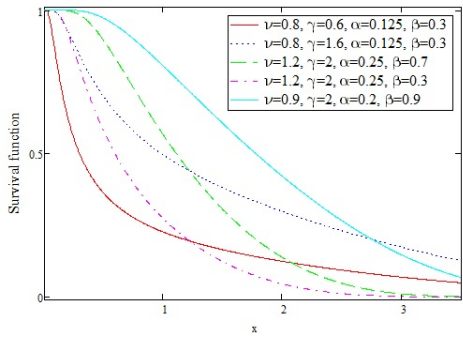

(a) $S(x)$

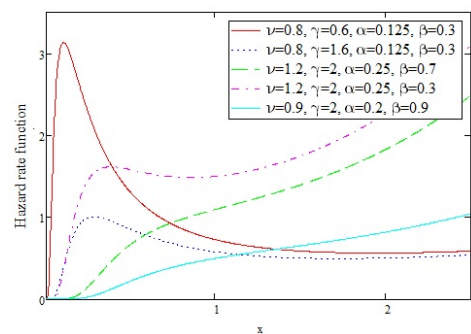

(b) $h(x)$

Fig. 2. The survival and hazard rate functions of the OGE-FWE for different values of parameters.

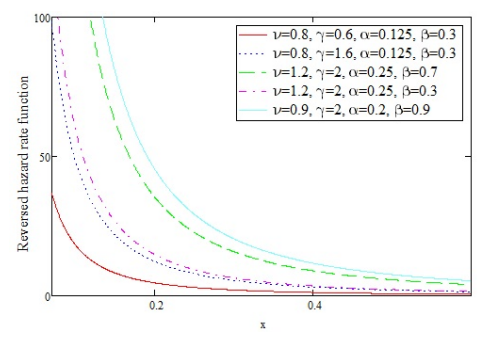

Fig. 3. The reversed hazard rate function of the OGE-FWE for different values of parameters.

\section{Statistical Properties}

In this section, we will study some statistical properties for the OGE-FWE distribution, specially quantile function and simulation median, the mode, moments, skewness and kurtosis.

\subsection{Quantile and median}

We determine the explicit formulas of the quantile and simulation median of the OGE-FWE distribution. The quantile $x_{q}$ of the $\operatorname{OGE}-\mathrm{FWE}(\vartheta, \gamma, \alpha, \beta)$ distribution is given by

$$
F\left(x_{q}\right)=P\left[x_{q} \leq q\right]=q, 0<q<1 .
$$


From Eq. (2.1), we have

$$
\left[1-e^{-\vartheta\left(e^{\alpha x_{q}-\frac{\beta}{x_{q}}}-1\right)}\right]^{\gamma}=q,
$$

we obtain $x_{q}$ by solving the following equation.

$$
\alpha x_{q}^{2}-k(q) x_{q}-\beta=0,
$$

where

$$
k(q)=\ln \left\{\ln \left[1-\frac{\ln \left(1-q^{\frac{1}{\gamma}}\right)}{\vartheta}\right]\right\} .
$$

So, the simulation of the OGE-FWE random variable is straightforward. If $\mathrm{U}$ is a uniform random variable on unit interval $(0,1)$. Then, by means of the inverse transformation method, we can obtain the random variable $X$ as follows

$$
X=\frac{k(u) \pm \sqrt{k(u)^{2}+4 \alpha \beta}}{2 \alpha} .
$$

Since the median of OGE-FWE distribution can be obtain by setting $q=0.5$ in Eq. (3.3).

\subsection{The mode}

In this subsection, we can obtain the mode of the OGE-FWE distribution by differentiating its probability density function pdf with respect to $x$ and equaling it to zero. The mode is the solution the following equation

$$
f^{\prime}(x)=0
$$

Since

$$
f(x ; \vartheta, \gamma, \alpha, \beta))=h(x ; \vartheta, \gamma, \alpha, \beta) S(x ; \vartheta, \gamma, \alpha, \beta) .
$$

Then from Eq. (3.5), we have

$$
\left[h^{\prime}(x ; \vartheta, \gamma, \alpha, \beta)-h^{2}(x ; \vartheta, \gamma, \alpha, \beta)\right] S(x ; \vartheta, \gamma, \alpha, \beta)=0,
$$

where $h(x ; \vartheta, \gamma, \alpha, \beta)$ is hazard rate function of OGE-FWE distribution Eq. (2.4), and $S(x ; \vartheta, \gamma, \alpha, \beta)$ is survival function of OGE-FWE Eq. (2.3).

It is difficult to get an analytic solution in $x$ to Eq. (3.6) in the general case. So, it has to be obtained by numerically methods.

\subsection{Skewness and Kurtosis}

In this subsection, we obtain the skewness and kurtosis based on quantile measures. The Moors Kurtosis is given by, [13]

$$
K_{u}=\frac{q_{(0.875)}-q_{(0.625)}-q_{(0.375)}+q_{(0.125)}}{q_{(0.75)}-q_{(0.25)}},
$$

and the Bowely's skewness based on quartiles is given by, [10] 


$$
S_{k}=\frac{q_{(0.75)}-2 q_{(0.5)}+q_{(0.25)}}{q_{(0.75)}-q_{(0.25)}}
$$

where $q_{(.)}$is quantile function.

\subsection{The Moments}

We derive the $r$ th moment for the OGE-FWE distribution in Theorem (3.1)

Theorem 3.1. If $X$ has $O G E-F W E(\vartheta, \gamma, \alpha, \beta)$ distribution, then The rth moments of random variable $X$, is given by

$$
\begin{aligned}
\mu_{r}^{\prime}= & \sum_{i=0}^{\infty} \sum_{j=0}^{\infty} \sum_{k=0}^{\infty} \sum_{\ell=0}^{\infty} \sum_{m=0}^{\infty} \frac{(-1)^{i+j+k+m} \gamma \vartheta^{j+1}(i+1)^{j}(j-k+1)^{\ell} \beta^{m}}{j ! \ell ! m !(\ell+1)^{r-2 m-1} \alpha^{r-m-1}} \times \\
& \left(\begin{array}{c}
\gamma-1 \\
i
\end{array}\right)\left(\begin{array}{l}
j \\
k
\end{array}\right)\left[\frac{\Gamma(r-m+1)}{\alpha(\ell+1)^{2}}+\beta \Gamma(r-m-1)\right] .
\end{aligned}
$$

Proof. We start with the well known distribution of the $r$ th moment of the random variable $X$ with probability density function $f(x)$ given by

$$
\mu_{r}^{\prime}=\int_{0}^{\infty} x^{r} f(x ; \vartheta, \gamma, \alpha, \beta) d x
$$

Substituting from Eq. (2.2) into Eq. (3.10) we get

$$
\mu_{r}^{\prime}=\int_{0}^{\infty} x^{r} \vartheta \gamma\left(\alpha+\frac{\beta}{x^{2}}\right) e^{\alpha x-\frac{\beta}{x}} e^{e^{\alpha x-\frac{\beta}{x}}} e^{-\vartheta\left(e^{e^{\alpha x-\frac{\beta}{x}}}-1\right)}\left[1-e^{-\vartheta\left(e^{e^{\alpha x-\frac{\beta}{x}}}-1\right)}\right]^{\gamma-1} d x
$$

since $0<\left[1-e^{-\vartheta\left(e^{e^{\alpha x-\frac{\beta}{x}}}-1\right)}\right]<1$ for $x>0$, the binomial series expansion of $\left[1-e^{-\vartheta\left(e^{e^{\alpha x-\frac{\beta}{x}}}-1\right)}\right]^{\gamma-1}$ yields

$$
\left[1-e^{-\vartheta\left(e^{e^{\alpha x-\frac{\beta}{x}}}-1\right)}\right]^{\gamma-1}=\sum_{i=0}^{\infty}(-1)^{i}\left(\begin{array}{c}
\gamma-1 \\
i
\end{array}\right) e^{-\vartheta i\left(e^{e^{\alpha x-\frac{\beta}{x}}}-1\right)}
$$

then we get

$$
\mu_{r}^{\prime}=\sum_{i=0}^{\infty}(-1)^{i}\left(\begin{array}{c}
\gamma-1 \\
i
\end{array}\right) \vartheta \gamma \int_{0}^{\infty} x^{r}\left(\alpha+\frac{\beta}{x^{2}}\right) e^{\alpha x-\frac{\beta}{x}} e^{e^{\alpha x-\frac{\beta}{x}}} e^{-\vartheta(i+1)\left(e^{\alpha x-\frac{\beta}{x}}-1\right)} d x,
$$

using series expansion

$$
e^{-\vartheta(i+1)\left(e^{e^{\alpha x-\frac{\beta}{x}}}-1\right)}=\sum_{j=0}^{\infty} \frac{(-1)^{j} \vartheta^{j}(i+1)^{j}}{j !}\left(e^{e^{\alpha x-\frac{\beta}{x}}}-1\right)^{j}
$$

we obtain

$$
\mu_{r}^{\prime}=\sum_{i=0}^{\infty} \sum_{j=0}^{\infty}(-1)^{i+j}\left(\begin{array}{c}
\gamma-1 \\
i
\end{array}\right) \frac{\gamma \vartheta^{j+1}(i+1)^{j}}{j !} \int_{0}^{\infty} x^{r}\left(\alpha+\frac{\beta}{x^{2}}\right) e^{\alpha x-\frac{\beta}{x}} e^{e^{\alpha x-\frac{\beta}{x}}}\left[e^{e^{\alpha x-\frac{\beta}{x}}}-1\right]^{j} d x
$$


Using series expansion

$$
\left(e^{e^{\alpha x-\frac{\beta}{x}}}-1\right)^{j}=\sum_{k=0}^{\infty}(-1)^{k}\left(\begin{array}{l}
j \\
k
\end{array}\right) e^{(j-k) e^{\alpha x-\frac{\beta}{x}}}
$$

hence

$$
\mu_{r}^{\prime}=\sum_{i=0}^{\infty} \sum_{j=0}^{\infty} \sum_{k=0}^{\infty}\left(\begin{array}{c}
\gamma-1 \\
i
\end{array}\right)\left(\begin{array}{l}
j \\
k
\end{array}\right) \frac{(-1)^{i+j+k} \gamma \vartheta^{j+1}(i+1)^{j}}{j !} \int_{0}^{\infty} x^{r}\left(\alpha+\frac{\beta}{x^{2}}\right) e^{\alpha x-\frac{\beta}{x}} e^{(j-k+1) e^{\alpha x-\frac{\beta}{x}}} d x
$$

using series expansion

$$
e^{(j-k+1) e^{\alpha x-\frac{\beta}{x}}}=\sum_{\ell=0}^{\infty} \frac{(j-k+1)^{\ell} e^{\ell\left(\alpha x-\frac{\beta}{x}\right)}}{\ell !}
$$

we obtain

$\mu_{r}^{\prime}=\sum_{i=0}^{\infty} \sum_{j=0}^{\infty} \sum_{k=0}^{\infty} \sum_{\ell=0}^{\infty}\left(\begin{array}{c}\gamma-1 \\ i\end{array}\right)\left(\begin{array}{l}j \\ k\end{array}\right) \frac{(-1)^{i+j+k} \gamma \vartheta^{j+1}(i+1)^{j}(j-k+1)^{\ell}}{j ! \ell !} \int_{0}^{\infty} x^{r}\left(\alpha+\frac{\beta}{x^{2}}\right) e^{(\ell+1)\left(\alpha x-\frac{\beta}{x}\right)} d x$

Using series expansion

$$
e^{-(\ell+1) \frac{\beta}{x}}=\sum_{m=0}^{\infty} \frac{(-\beta)^{m}(\ell+1)^{m}}{m !} x^{-m}
$$

gives

$$
\begin{aligned}
\mu_{r}^{\prime}= & \sum_{i=0}^{\infty} \sum_{j=0}^{\infty} \sum_{k=0}^{\infty} \sum_{\ell=0}^{\infty} \sum_{m=0}^{\infty} \frac{(-1)^{i+j+k+m} \gamma \vartheta^{j+1}(i+1)^{j}(j-k+1)^{\ell} \beta^{m}(\ell+1)^{m}}{j ! \ell ! m !}\left(\begin{array}{c}
\gamma-1 \\
i
\end{array}\right)\left(\begin{array}{l}
j \\
k
\end{array}\right) \times \\
& \int_{0}^{\infty} x^{r-m}\left(\alpha+\frac{\beta}{x^{2}}\right) e^{(\ell+1) \alpha x} d x, \\
= & \sum_{i=0}^{\infty} \sum_{j=0}^{\infty} \sum_{k=0}^{\infty} \sum_{\ell=0}^{\infty} \sum_{m=0}^{\infty} \frac{(-1)^{i+j+k+m} \gamma \vartheta^{j+1}(i+1)^{j}(j-k+1)^{\ell} \beta^{m}(\ell+1)^{m}}{j ! \ell ! m !}\left(\begin{array}{c}
\gamma-1 \\
i
\end{array}\right)\left(\begin{array}{c}
j \\
k
\end{array}\right) \times \\
& {\left[\int_{0}^{\infty} \alpha x^{r-m} e^{(\ell+1) \alpha x} d x+\int_{0}^{\infty} \beta x^{r-m-2} e^{(\ell+1) \alpha x} d x\right], }
\end{aligned}
$$

by using the definition of gamma function in the form, [26],

$$
\Gamma(z)=x^{z} \int_{0}^{\infty} e^{t x} t^{z-1} d t, z, x,>0 .
$$

Finally, we obtain the $r$ th moment of OGE-FWE distribution in the form

$$
\begin{aligned}
\mu_{r}^{\prime}= & \sum_{i=0}^{\infty} \sum_{j=0}^{\infty} \sum_{k=0}^{\infty} \sum_{\ell=0}^{\infty} \sum_{m=0}^{\infty} \frac{(-1)^{i+j+k+m} \gamma \vartheta^{j+1}(i+1)^{j}(j-k+1)^{\ell} \beta^{m}(\ell+1)^{m}}{j ! \ell ! m !}\left(\begin{array}{c}
\gamma-1 \\
i
\end{array}\right)\left(\begin{array}{l}
j \\
k
\end{array}\right) \times \\
& {\left[\frac{\Gamma(r-m+1)}{\alpha^{r-m}(\ell+1)^{r-m+1}}+\frac{\beta \Gamma(r-m-1)}{\alpha^{r-m-1}(\ell+1)^{r-m-1}}\right] . }
\end{aligned}
$$

This completes the proof. 


\section{The Moment Generating Function}

The moment generating function (mgf) of the OGE-FWE distribution is given by Theorem (4.1).

Theorem 4.1. The moment generating function (mgf) of OGE-FWE distribution is given by

$$
\begin{aligned}
M_{X}(t)= & \sum_{r=0}^{\infty} \sum_{i=0}^{\infty} \sum_{j=0}^{\infty} \sum_{k=0}^{\infty} \sum_{\ell=0}^{\infty} \sum_{m=0}^{\infty} \frac{(-1)^{i+j+k+m} \gamma \vartheta^{j+1}(i+1)^{j}(j-k+1)^{\ell} \beta^{m} t^{r}}{j ! \ell ! m ! r ! \alpha^{r-m-1}(\ell+1)^{r-2 m-1}} \\
& \times\left(\begin{array}{c}
\gamma-1 \\
i
\end{array}\right)\left(\begin{array}{l}
j \\
k
\end{array}\right)\left[\frac{\Gamma(r-m+1)}{\alpha(\ell+1)^{2}}+\beta \Gamma(r-m-1)\right] .
\end{aligned}
$$

Proof. The moment generating function $M_{X}(t)$ of the random variable $X$ with probability density function $f(x)$ is given by

$$
M_{X}(t)=\int_{0}^{\infty} e^{t x} f(x ; \vartheta, \gamma, \alpha, \beta) d x
$$

Using series expansion of $e^{t x}$, we obtain

$$
M_{X}(t)=\sum_{r=0}^{\infty} \frac{t^{r}}{r !} \int_{0}^{\infty} x^{r} f(x) d x=\sum_{r=0}^{\infty} \frac{t^{r}}{r !} \mu_{r}^{\prime}
$$

Substituting from (3.9) into (4.3), we obtain

$$
\begin{aligned}
M_{X}(t)= & \sum_{r=0}^{\infty} \sum_{i=0}^{\infty} \sum_{j=0}^{\infty} \sum_{k=0}^{\infty} \sum_{\ell=0}^{\infty} \sum_{m=0}^{\infty} \frac{(-1)^{i+j+k+m} \gamma \vartheta^{j+1}(i+1)^{j}(j-k+1)^{\ell} \beta^{m} t^{r}}{j ! \ell ! m ! r ! \alpha^{r-m-1}(\ell+1)^{r-2 m-1}} \\
& \times\left(\begin{array}{c}
\gamma-1 \\
i
\end{array}\right)\left(\begin{array}{l}
j \\
k
\end{array}\right)\left[\frac{\Gamma(r-m+1)}{\alpha(\ell+1)^{2}}+\beta \Gamma(r-m-1)\right] .
\end{aligned}
$$

This completes the proof.

\section{Order Statistics}

In this section, we derive closed form expressions for the PDFs of the $r$ th order statistic of the OGEFWE distribution. Let $X_{1: n}, X_{2: n}, \cdots, X_{n: n}$ denote the order statistics obtained from a random sample $X_{1}, X_{2}, \cdots, X_{n}$ which taken from a continuous population with cumulative distribution function cdf $F(x ; \varphi)$ and probability density function pdf $f(x ; \varphi)$, then the pdf of $X_{r: n}$ is given as follows

$$
f_{r: n}(x ; \varphi)=\frac{1}{B(r, n-r+1)}[F(x ; \varphi)]^{r-1}[1-F(x ; \varphi)]^{n-r} f(x ; \varphi),
$$

where $f(x ; \varphi)$ and $F(x ; \varphi)$ are the pdf and cdf of OGE-FWE $(\varphi)$ distribution given by Eq. (2.2) and Eq. (1.7) respectively, $\varphi=(\vartheta, \gamma, \alpha, \beta)$ and $B(.,$.$) is the Beta function, also we define first order$ statistics $X_{1: n}=\min \left(X_{1}, X_{2}, \cdots, X_{n}\right)$ and the last order statistics as $X_{n: n}=\max \left(X_{1}, X_{2}, \cdots, X_{n}\right)$. Since 
$0<F(x ; \varphi)<1$ for $x>0$, we can use the binomial expansion of $[1-F(x ; \varphi)]^{n-r}$ as follows

$$
[1-F(x ; \varphi)]^{n-r}=\sum_{i=0}^{n-r}\left(\begin{array}{c}
n-r \\
i
\end{array}\right)(-1)^{i}[F(x ; \varphi)]^{i}
$$

Substituting from Eq. (5.2) into Eq. (5.1), we obtain

$$
f_{r: n}(x ; \vartheta, \gamma, \alpha, \beta)=\sum_{i=0}^{n-r} \frac{(-1)^{i} n !}{i !(r-1) !(n-r-i) !} f(x ; \varphi)[F(x ; \varphi)]^{i+r-1} .
$$

Substituting from Eq. (2.1) and Eq. (2.2) into Eq. (5.3), we obtain the probability density function for $r$ th order statistics.

Relation (5.3) show that $f_{r: n}(x ; \varphi)$ is the weighted average of the OGE-FWE distribution with different shape parameters.

\section{Parameters Estimation}

In this section, point and interval estimation of the unknown parameters of the OGE-FWE distribution are derived by using the maximum likelihood method based on a complete sample.

\subsection{Maximum Likelihood Estimation:}

Let $x_{1}, x_{2}, \cdots, x_{n}$ denote a random sample of complete data from the OGE-FWE distribution. The Likelihood function is given as

$$
L=\prod_{i=1}^{n} f\left(x_{i} ; \vartheta, \gamma, \alpha, \beta\right)
$$

substituting from (2.2) into (6.1), we have

$$
L=\prod_{i=1}^{n} \vartheta \gamma\left(\alpha+\frac{\beta}{x_{i}^{2}}\right) e^{\alpha x_{i}-\frac{\beta}{x_{i}}} e^{e^{\alpha x_{i}-\frac{\beta}{x_{i}}}} e^{-\vartheta\left[e^{e^{\alpha x_{i}-\frac{\beta}{x_{i}}}}-1\right]}\left[1-e^{-\vartheta\left[e^{e^{\alpha x_{i}-\frac{\beta}{x_{i}}}}-1\right]}\right]^{\gamma-1}
$$

The log-likelihood function is

$$
\begin{aligned}
\mathscr{L}= & n \ln (\vartheta \gamma)+\sum_{i=1}^{n} \ln \left(\alpha+\frac{\beta}{x_{i}^{2}}\right)+\sum_{i=1}^{n}\left(\alpha x_{i}-\frac{\beta}{x_{i}}\right)+\sum_{i=1}^{n} e^{\alpha x_{i}-\frac{\beta}{x_{i}}}-\vartheta \sum_{i=1}^{n}\left(e^{e^{\alpha x_{i}-\frac{\beta}{x_{i}}}}-1\right) \\
& \left.+(\gamma-1) \sum_{i=1}^{n} \ln \left[1-e^{-\vartheta\left(e^{e} x^{\alpha x_{i}-\frac{\beta}{x_{i}}}\right.}-1\right)\right] .
\end{aligned}
$$

The maximum likelihood estimation of the parameters $(\vartheta, \gamma, \alpha, \beta)$ are obtained by differentiated the $\log$-likelihood function $\mathscr{L}$ with respect to the parameters $\vartheta, \gamma, \alpha$ and $\beta$ and setting the result to zero, we have the following normal equations. 


$$
\begin{aligned}
& \frac{\partial \mathscr{L}}{\partial \vartheta}=\frac{n}{\vartheta}-\sum_{i=1}^{n}\left(e^{e^{\alpha x_{i}-\frac{\beta}{x_{i}}}}-1\right)+(\gamma-1) \sum_{i=1}^{n} \frac{e^{e^{\alpha x_{i}-\frac{\beta}{x_{i}}}}-1}{e^{\vartheta\left(e^{e^{\alpha x_{i}-\frac{\beta}{x_{i}}}}-1\right)}-1}=0 \\
& \left.\frac{\partial \mathscr{L}}{\partial \gamma}=\frac{n}{\gamma}+\sum_{i=1}^{n} \ln \left(1-e^{-\vartheta\left(e^{\alpha x_{i}-\frac{\beta}{x_{i}}}\right.}-1\right)\right)=0 \\
& \frac{\partial \mathscr{L}}{\partial \alpha}=\sum_{i=1}^{n} \frac{x_{i}^{2}}{\beta+\alpha x_{i}^{2}}+\sum_{i=1}^{n} x_{i}+\sum_{i=1}^{n} x_{i} e^{\alpha x_{i}-\frac{\beta}{x_{i}}}-\vartheta \sum_{i=1}^{n} x_{i} \mathscr{D}_{i}+\vartheta(\gamma-1) \sum_{i=1}^{n} \frac{x_{i} \mathscr{D}_{i}}{e^{\vartheta\left(e^{\alpha x_{i}-\frac{\beta}{x_{i}}}-1\right)}-1}=0 \\
& \frac{\partial \mathscr{L}}{\partial \beta}=\sum_{i=1}^{n} \frac{1}{\beta+\alpha x_{i}^{2}}-\sum_{i=1}^{n} \frac{1}{x_{i}}-\sum_{i=1}^{n} \frac{1}{x_{i}} e^{\alpha x_{i}-\frac{\beta}{x_{i}}}+\vartheta \sum_{i=1}^{n} \frac{\mathscr{D}_{i}}{x_{i}}-\vartheta(\gamma-1) \sum_{i=1}^{n} \frac{\mathscr{D}_{i}}{x_{i}\left[e^{\vartheta\left(e^{e^{\alpha x_{i}-\frac{\beta}{x_{i}}}}-1\right)}-1\right]}=0,
\end{aligned}
$$

where $\mathscr{D}_{i}=\exp \left\{\alpha x_{i}-\frac{\beta}{x_{i}}+e^{\alpha x_{i}-\frac{\beta}{x_{i}}}\right\}$. The MLEs can be obtained by solving the equations, (6.3)(6.6), numerically for $\vartheta, \gamma, \alpha$ and $\beta$.

\subsection{Asymptotic confidence bounds}

In this section, we derive the asymptotic confidence intervals of these parameters when $\vartheta, \gamma, \alpha>0$ and $\beta>0$ as the MLEs of the unknown parameters $\vartheta, \gamma, \alpha>0$ and $\beta>0$ can not be obtained in closed forms, by using variance covariance matrix $I^{-1}$ see [12], where $I^{-1}$ is the inverse of the observed information matrix which defined as follows

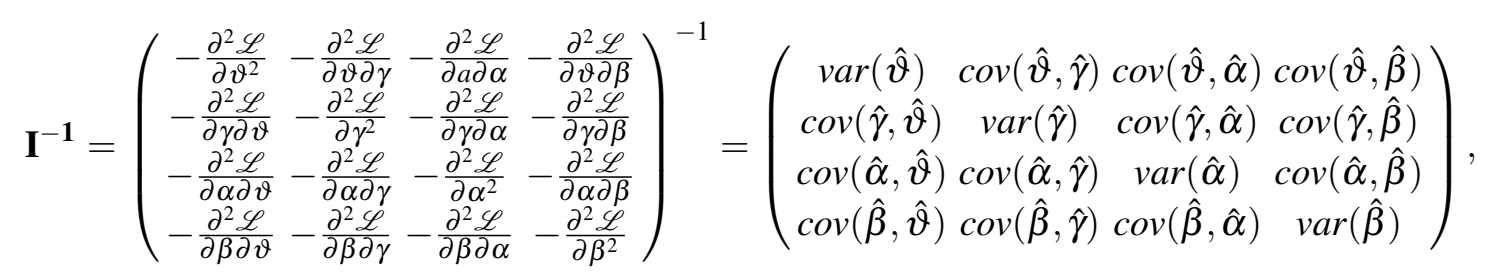

where

$$
\begin{aligned}
\frac{\partial^{2} \mathscr{L}}{\partial \vartheta^{2}} & =-\frac{n}{\vartheta^{2}}-(\gamma-1) \sum_{i=1}^{n} e^{\vartheta\left(e^{e^{\alpha x_{i}-\frac{\beta}{x_{i}}}}-1\right)} \mathscr{A}_{i}^{2}, & \frac{\partial^{2} \mathscr{L}}{\partial \vartheta \partial \gamma} & =\sum_{i=1}^{n} \mathscr{A}_{i} \\
\frac{\partial^{2} \mathscr{L}}{\partial \vartheta \partial \alpha} & =-\sum_{i=1}^{n} x_{i} \mathscr{D}_{i}+(\gamma-1) \sum_{i=1}^{n} x_{i} \mathscr{B}_{i}, & \frac{\partial^{2} \mathscr{L}}{\partial \vartheta \partial \beta} & =\sum_{i=1}^{n} \frac{\mathscr{D}_{i}}{x_{i}}-(\gamma-1) \sum_{i=1}^{n} \frac{\mathscr{B}_{i}}{x_{i}} \\
\frac{\partial^{2} \mathscr{L}}{\partial \gamma^{2}} & =-\frac{n}{\gamma^{2}}, & \frac{\partial^{2} \mathscr{L}}{\partial \gamma \partial \alpha} & =\vartheta \sum_{i=1}^{n} \frac{x_{i} \mathscr{D}_{i}}{e^{\vartheta\left(e^{\alpha x_{i}-\frac{\beta}{x_{i}}}-1\right)}-1}
\end{aligned}
$$




$$
\begin{aligned}
\frac{\partial^{2} \mathscr{L}}{\partial \gamma \partial \beta} & =-\vartheta \sum_{i=1}^{n} \frac{\mathscr{D}_{i}}{x_{i}\left[e^{\vartheta\left(e^{\alpha x_{i}-\frac{\beta}{x_{i}}}-1\right)}-1\right]} \\
\frac{\partial^{2} \mathscr{L}}{\partial \alpha^{2}} & =-\sum_{i=1}^{n} \frac{x_{i}^{4}}{\left(\beta+\alpha x_{i}^{2}\right)^{2}}+\sum_{i=1}^{n} x_{i}^{2} e^{\alpha x_{i}-\frac{\beta}{x_{i}}}-\vartheta \sum_{i=1}^{n} x_{i}^{2} \mathscr{D}_{i}\left[e^{\alpha x_{i}-\frac{\beta}{x_{i}}}+1\right]+\vartheta(\gamma-1) \sum_{i=1}^{n} x_{i}^{2} \mathscr{K}_{i}(6.1) \\
\frac{\partial^{2} \mathscr{L}}{\partial \alpha \partial \beta} & =-\sum_{i=1}^{n} \frac{x_{i}^{2}}{\left(\beta+\alpha x_{i}^{2}\right)^{2}}-\sum_{i=1}^{n} e^{\alpha x_{i}-\frac{\beta}{x_{i}}}+\vartheta \sum_{i=1}^{n} \mathscr{D}_{i}\left[e^{\alpha x_{i}-\frac{\beta}{x_{i}}}+1\right]-\vartheta(\gamma-1) \sum_{i=1}^{n} \mathscr{K}_{i} \\
\frac{\partial^{2} \mathscr{L}}{\partial \beta^{2}} & =-\sum_{i=1}^{n} \frac{1}{\left(\beta+\alpha x_{i}^{2}\right)^{2}}+\sum_{i=1}^{n} \frac{e^{\alpha x_{i}-\frac{\beta}{x_{i}}}}{x_{i}^{2}}-\vartheta \sum_{i=1}^{n} \frac{\mathscr{D}_{i}\left[e^{\alpha x_{i}-\frac{\beta}{x_{i}}}+1\right]}{x_{i}^{2}}+\vartheta(\gamma-1) \sum_{i=1}^{n} \frac{\mathscr{K}_{i}}{x_{i}^{2}}
\end{aligned}
$$

where

$$
\begin{aligned}
\mathscr{A}_{i} & =\left[e^{e^{\alpha x_{i}-\frac{\beta}{x_{i}}}}-1\right]\left[e^{\vartheta\left(e^{\alpha x_{i}-\frac{\beta}{x_{i}}}-1\right)}-1\right]^{-1}, \\
\mathscr{B}_{i} & \left.=\mathscr{D}_{i}\left[e^{\vartheta\left(e^{\alpha x_{i}-\frac{\beta}{x_{i}}}\right.}-1\right)\left(1-\vartheta\left(e^{e^{\alpha x_{i}-\frac{\beta}{x_{i}}}}-1\right)\right)-1\right]\left[e^{\vartheta\left[e^{e e^{\alpha x_{i}-\frac{\beta}{x_{i}}}}-1\right]}-1\right]^{-2}, \\
\mathscr{K}_{i} & =\mathscr{D}_{i}\left[\left(e^{\vartheta\left(e^{\alpha x_{i}-\frac{\beta}{x_{i}}}-1\right)}-1\right)\left(e^{\alpha x_{i}-\frac{\beta}{x_{i}}}+1\right)-\vartheta \mathscr{D}_{i} e^{\vartheta\left(e^{\alpha x_{i}-\frac{\beta}{x_{i}}}-1\right)}\right]\left[e^{\vartheta\left(e^{\alpha x_{i}-\frac{\beta}{x_{i}}}-1\right)}-1\right]^{-2} .
\end{aligned}
$$

We can obtain the $(1-\delta) 100 \%$ confidence intervals of the parameters $\vartheta, \gamma, \alpha$ and $\beta$ by using variance matrix as in the following forms

$$
\hat{\vartheta} \pm Z_{\frac{\delta}{2}} \sqrt{\operatorname{var}(\hat{\vartheta})}, \quad \hat{\gamma} \pm Z_{\frac{\delta}{2}} \sqrt{\operatorname{var}(\hat{\gamma})}, \quad \hat{\alpha} \pm Z_{\frac{\delta}{2}} \sqrt{\operatorname{var}(\hat{\alpha})}, \quad \hat{\beta} \pm Z_{\frac{\delta}{2}} \sqrt{\operatorname{var}(\hat{\beta})}
$$

where $Z_{\frac{\delta}{2}}$ is the upper $\left(\frac{\delta}{2}\right)$-th percentile of the standard normal distribution.

\section{Application}

In this section, we will analysis of a real data set using the OGE-FWE $(\vartheta, \gamma, \alpha, \beta)$ model and compare it with the other fitted models like a flexible Weibull extension distributions using Kolmogorov Smirnov (K-S) statistic, as well as Akaike information criterion(AIC), [?], Akaike Information Citerion with correction (AICC), Bayesian information criterion (BIC), Hannan-Quinn information criterion (HQIC) and Schwarz information criterion (SIC) values, [21]. The data have been obtained from [18], it is for the time between failures (thousands of hours) of secondary reactor pumps, Table 1 .

Table 1 . Time between failures of secondary reactor pumps.

\begin{tabular}{llllllll}
\hline 2.160 & 0.746 & 0.402 & 0.954 & 0.491 & 6.560 & 4.992 & 0.347 \\
0.150 & 0.358 & 0.101 & 1.359 & 3.465 & 1.060 & 0.614 & 1.921 \\
4.082 & 0.199 & 0.605 & 0.273 & 0.070 & 0.062 & 5.320 & \\
\hline
\end{tabular}


Table 2. MLEs and K-S of parameters for secondary reactor pumps.

\begin{tabular}{lcccccc}
\hline Model & $\hat{\alpha}$ & $\hat{\beta}$ & $\hat{\lambda}$ & $\hat{\vartheta}$ & $\hat{\gamma}$ & K-S \\
\hline OGE-FWE & 0.2380 & 2.0700 & - & 0.069 & 0.113 & 0.0760 \\
Flexible Weibull & 0.0207 & 2.5875 & - & - & - & 0.1342 \\
Weibull & 0.8077 & 13.9148 & - & - & - & 0.1173 \\
Modified Weibull & 0.1213 & 0.7924 & 0.0009 & - & - & 0.1188 \\
Reduced Additive Weibull & 0.0070 & 1.7292 & 0.0452 & - & - & 0.1619 \\
Extended Weibull & 0.4189 & 1.0212 & 10.2778 & - & - & 0.1057 \\
\hline
\end{tabular}

Table 3. Log-likelihood, AIC, AICC, BIC, HQIC and SIC values of models fitted.

\begin{tabular}{lcccccc}
\hline Model & L & AIC & AICC & BIC & HQIC & SIC \\
\hline OGE-FEW & -29.2980 & 66.5960 & 68.8182 & 71.1380 & 10.5590 & 71.1380 \\
Flexible Weibull & -83.3424 & 170.6848 & 171.2848 & 172.9558 & 12.5416 & 172.9558 \\
Weibull & -85.4734 & 174.9468 & 175.5468 & 177.2178 & 12.5915 & 177.2178 \\
Modified Weibull & -85.4677 & 176.9354 & 178.1986 & 180.3419 & 12.6029 & 180.3419 \\
Reduced additive Weibull & -86.0728 & 178.1456 & 179.4088 & 181.5521 & 12.6168 & 181.5521 \\
Extended Weibull & -86.6343 & 179.2686 & 180.5318 & 182.6751 & 12.6296 & 182.6751 \\
\hline
\end{tabular}

Table 2 gives MLEs of parameters of the OGE-FWE and K-S Statistics. The values of the loglikelihood functions, AIC, AICC, BIC, HQIC, and SIC are in Table 3.

Substituting the MLEs of the unknown parameters $\vartheta, \gamma, \alpha, \beta$ into (6.7), we obtain estimation of the variance covariance matrix as the following

$$
I_{0}^{-1}=\left(\begin{array}{cccr}
6.773 \times 10^{-3} & 4.189 \times 10^{-4} & -3.945 \times 10^{-3} & -1.65 \times 10^{-3} \\
4.189 \times 10^{-4} & 4.308 \times 10^{-4} & -4.871 \times 10^{-4} & -0.013 \\
-3.945 \times 10^{-3} & -4.871 \times 10^{-4} & 3.343 \times 10^{-3} & 0.017 \\
-1.65 \times 10^{-3} & -0.013 & 0.017 & 0.806
\end{array}\right) \text {. }
$$

The approximate $95 \%$ two sided confidence intervals of the unknown parameters $\vartheta, \gamma, \alpha$ and $\beta$ are $[0,0.230],[0.072,0.154],[0.125,0.351]$ and $[0.31,3.83]$, respectively.

The nonparametric estimate of the survival function $S(x)$ using the Kaplan-Meier method and its fitted parametric estimations when the distribution is assumed to be OGE-FWE, FW, W, MW, RAW and EW are computed and plotted in Figure 4.

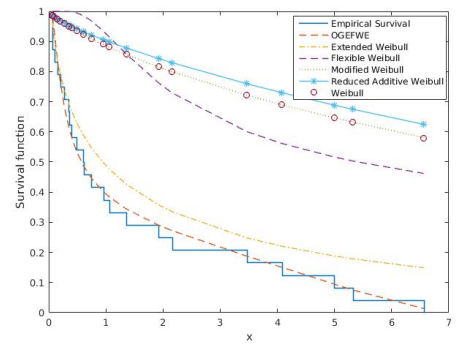

Fig. 4. The Kaplan-Meier estimate of the survival function for the data. 
Figure 5 gives the form of the hazard rate $h(x)$ and cumulative density function cdf for the OGEFWE, FW, W, MW, RAW and EW which are used to fit the data after the unknown parameters included in each distribution are replaced by their MLEs.

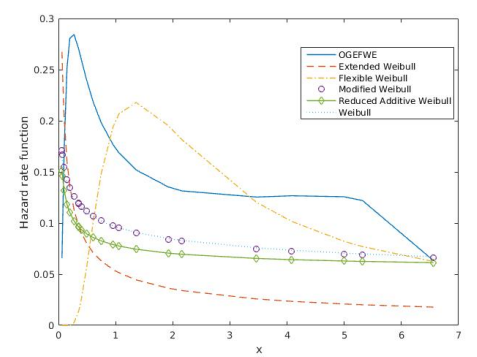

(a) $h(t)$

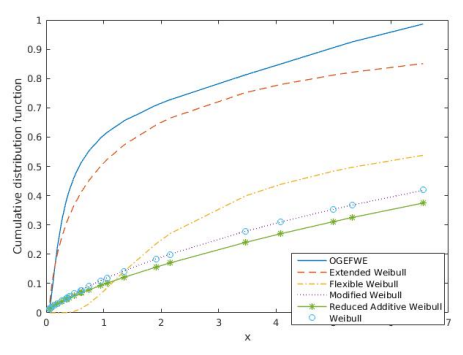

(b) cdf

Fig. 5. The Fitted hazard rate and cumulative distribution function for the data.

We find that the OGE-FWE distribution with the four - parameters provides a better fit than the previous new modified a flexible Weibull extension distribution(FWE) which was the best in [4]. It has the largest likelihood, and the smallest AIC, AICC, BIC, HQIC and SIC values among those considered in this paper.

\section{Conclusions}

We proposed a new distribution, based on odd generalized exponential family distributions, this distribution is named the odd generalized exponential flexible Weibull extension OGE-FWE distribution. Some its statistical properties are studied. The maximum likelihood method is used for estimating the parameters model. Finally, we introduce an application using real data. We show that the OGE-FWE distribution fits certain well known data sets better than existing modifications of the Weibull and flexible Weibull extension distributions.

\section{References}

[1] H. Akaike, A new look at the statistical model identification, IEEE Transactions on Automatic Control AC-19 (1974) 716-23.

[2] S.J. Almalki and J. Yuan, The new modified Weibull distribution, Reliability Engineering and System Safety 111 (2013) 164-170.

[3] E. K. AL-Hussaini and M. Ahsanullah, Exponentiated Distributions. Atlantis-Press, Paris, France (2015).

[4] M.S. Bebbington, C.D. Lai and R. Zitikis, A flexible Weibull extension. Reliability Engineering \& System Safety 92(6) (2007), 719-26.

[5] M. Carrasco, E.M. Ortega and G.M. Cordeiro, A generalized modified Weibull distribution for lifetime modeling, Computational Statistics and Data Analysis 53(2) (2008) 450-62.

[6] G.M. Cordeiro, E.M. Ortega and S. Nadarajah, The Kumaraswamy Weibull distribution with application to failure data. Journal of the Franklin Institute 347 (2010) 1399-429.

[7] M. A. El-Damcese, A. Mustafa, B. S. El-Desouky and M. E. Mustafa, The odd generalized exponential gompertz, arXiv preprint arXiv:1507.06400 (2015).

[8] F. Famoye, C. Lee and O. Olumolade, The beta-Weibull distribution, Journal of Statistical Theory and Applications 4( 2) (2005) 121-36. 
[9] R.D. Gupta and D. Kundu, Generalized exponential distribution: Existing results and some recent developments, Journal of Statistical Planning and Inference 137(11) (1999) 3537-3547.

[10] J. Kenney and E. Keeping, Mathematics of Statistics, Princeton 1 (1962).

[11] C.D. Lai, M. Xie and D.N.P. Murthy, A modified Weibull distributions, IEEE Transactions on Reliability 52(1) (2003), 33-7.

[12] J. F. Lawless, Statistical Models and Methods for Lifetime Data, John Wiley and Sons, New York 20 (2003), 1108-1113.

[13] J.J.A. Moors, A quantile alternative for kurtosis, The Statistician 37 (1998), 25-32.

[14] G.S. Mudholkar and D.K. Srivastava, Exponentiated Weibull family for analyzing bathtub failure-rate data, IEEE Transactions on Reliability 42(2) (1993) 299-302.

[15] D. N. P. Murthy, M. Xie and R. Jiang, Weibull Models, John Wiley and Sons, New York (2003).

[16] S. Nadarajah, G.M. Cordeiro and E.M.M. Ortega, General results for the beta-modified Weibull distribution, Journal of Statistical Computation and Simulation 81(10) (2011), 1211-32.

[17] H. Pham and C.D. Lai, On recent generalizations of the Weibull distribution, IEEE Transactions on Reliability 56 (2007) 454-8.

[18] M. Salman, Suprawhardana and S. Prayoto, Total time on test plot analysis for mechanical components of the RSG-GAS reactor, Atom Indones 25(2) (1999), 155-61.

[19] A.M. Sarhan and M. Zaindin, Modified Weibull distribution, Applied Sciences 11 (2009) 123-136.

[20] A.M. Sarhan and J. Apaloo, Exponentiated modified Weibull extension distribution, Reliability Engineering and System Safety 112 (2013) 137-144.

[21] G. Schwarz, Estimating the dimension of a model, Annals of Statistics 6 (1978) 461-4.

[22] G.O. Silva, E.M. Ortega and G.M. Cordeiro, The beta modified Weibull distribution, Lifetime Data Analysis 16 (2010), 409-30.

[23] N. Singla, K. Jain and S. S. Kumar, The beta generalized Weibull distribution: properties and applications, Reliability Engineering \& System Safety 102 (2012) 5-15.

[24] M. H. Tahir, G. M. Cordeiro, M. Alizadeh, M. Mansoor, M. Zubair and G. G. Hamedani, The odd generalized exponential family of distributions with applications, Journal of Statistical Distributions and Applications 2(1) (2015) 1-28.

[25] M. Xie and C.D. Lai, Reliability analysis using an additive Weibull model with bathtub-shaped failure ratefunction, Reliability Engineering System Safety 52 (1995) 87-93.

[26] D. Zwillinger, Table of integrals, series, and products, Elsevier (2014).

[27] W.A. Weibull, Statistical distribution function of wide applicability, Journal of Applied Mechanics 18 (1951) 293-6. 